\title{
$k$-cut model for the Brownian continuum random tree
}

\author{
Minmin Wang*
}

\begin{abstract}
To model the destruction of a resilient network, Cai, Holmgren, Devroye and Skerman introduced the $k$-cut model on a random tree, as an extension to the classic problem of cutting down random trees. Berzunza, Cai and Holmgren later proved that the total number of cuts in the $k$-cut model to isolate the root of a Galton-Watson tree with a finite-variance offspring law and conditioned to have $n$ nodes, when divided by $n^{1-1 / 2 k}$, converges in distribution to some random variable defined on the Brownian CRT. We provide here a direct construction of the limit random variable, relying upon the Aldous-Pitman fragmentation process and a deterministic time change.
\end{abstract}

Keywords: continuum random tree; Brownian excursion; random cut on random tree. MSC2020 subject classifications: 60C05; 60G18; 60G55.

Submitted to ECP on July 24, 2020, final version accepted on June 30, 2021.

Supersedes arXiv: 2007.11080.

\section{Introduction}

Let $k \in \mathbb{N}$ and let $T$ be a rooted tree. The following procedure is considered by Cai, Holmgren, Devroye and Skerman [9]. To each vertex $v$ of $T$, we associate an independent Poisson process $N_{v}=\left(N_{v}(t)\right)_{t \geq 0}$ of rate 1. Imagine that each time $N_{v}$ increases, the vertex $v$ is "cut" once; after it receives $k$ "cuts", it is removed along with all the adjacent edges. The procedure ends when the root is removed. We are interested in the total number of "cuts" falling on the subtree containing the root, denoted as $X_{k}(T)$. Let us observe that for $k=1$, the above procedure reduces to the classic problem of cutting down random trees introduced by Meir and Moon [14]; see in particular [12, 2, 5, 1, 7, 10] for some recent progress on the classical version. The current extension, on the other hand, can be seen as a simple model for attacks on computer networks with resilience ([9]).

Let $\xi=(\xi(p))_{p \geq 0}$ be a probability measure on the set of non negative integers which satisfies

$$
\sum_{p \geq 1} p \xi(p)=1, \quad \text { and } \quad 0<\sigma:=\left(\sum_{p \geq 2} p(p-1) \xi(p)\right)^{1 / 2}<\infty .
$$

For $n \geq 1$, let $T_{n}$ be a Galton-Watson tree with offspring distribution $\xi$ conditioned on having $n$ vertices. Berzunza, Cai and Holmgren show in [6] that

$$
\left(\frac{\sigma}{\sqrt{n}} T_{n}, \frac{X_{k}\left(T_{n}\right)}{\sigma^{\frac{1}{k}} n^{1-\frac{1}{2 k}}}\right) \underset{n \rightarrow \infty}{\stackrel{d}{\longrightarrow}}\left(\mathcal{T}, Z_{k}\right),
$$

\footnotetext{
${ }^{*}$ Department of Mathematics, University of Sussex, Falmer, Brighton BN1 9QH, United Kingdom. E-
} mail: minmin.wang@sussex.ac.uk 
where $\mathcal{T}$ is the so-called Brownian Continuum Random Tree, and $Z_{k}$ is a non degenerate random variable whose distribution is characterised via its moments. Note that the convergence of $\frac{\sigma}{\sqrt{n}} T_{n}$ to $\mathcal{T}$, due to Aldous [3], is well known and takes place in the weak topology of the Gromov-Hausdorff space. We defer the formal definitions of these objects till a later point. Let us also point out that the joint convergence in (1.1) generalises an earlier result for $k=1$ by Janson [12].

In the case $k=1$, it is also known that $Z_{1}$ can be explicitly written as a functional of the so-called Aldous-Pitman fragmentation process, thanks to the works of AddarioBerry, Broutin \& Holmgren [2], Bertoin \& Miermont [5], Abraham \& Delmas [1]. In this work, we extend this construction of $Z_{1}$ to the general setting of $k \geq 1$, thus answering a question in [6] on the construction of $Z_{k}$. To that end, let us start with a brief introduction to the Aldous-Pitman fragmentation process.

The Aldous-Pitman fragmentation process can be viewed as the analogue of the 1-cut model for the Brownian continuum random tree (CRT). First, we need to construct this CRT. Let us take $\mathrm{e}=\left(e_{s}\right)_{0 \leq s \leq 1}$, where $\frac{1}{2} \mathrm{e}$ is distributed as the standard normalised Brownian excursion of duration 1 . For $s, t \in[0,1]$, define

$$
d(s, t)=e_{s}+e_{t}-2 b(s, t), \quad \text { where } \quad b(s, t)=\min _{s \wedge t \leq u \leq s \vee t} e_{u}
$$

It turns out the function $d$ is non-negative, symmetric and satisfies the triangle inequality. To turn it into a metric, let $s \sim t$ if and only if $d(s, t)=0$. Then $d$ defines a metric on the quotient space $\mathcal{T}:=[0,1] / \sim$, which we still denote as $d$. In the sequel, we will refer to the (random) metric space $(\mathcal{T}, d)$ as the Brownian CRT. Note that it has "tree-like" features: each pair of points in $\mathcal{T}$, say $x$ and $y$, is joined by a unique path, denoted as $\llbracket x, y \rrbracket$, which turns out to be a geodesic. Metric spaces with such properties are called R-trees. Interested readers are referred to Evans [11] and Le Gall [13] for more background on R-trees and CRT.

Let us also introduce the following notation on $(\mathcal{T}, d)$ which will be useful later. We denote by $p:[0,1] \rightarrow \mathcal{T}$ the canonical projection which sends every $t \in[0,1]$ to its equivalence class with respect to $\sim$. The $\operatorname{root}$ of $(\mathcal{T}, d)$ is then the point $\rho=p(0)=p(1)$. In addition, the map $p$ also induces a probability measure on $\mathcal{T}$ : the mass measure, denoted as $\mu$, is the push-forward of the uniform measure on $[0,1]$ by $p$. On the other hand, the length measure $\ell$ is a $\sigma$-finite measure on $\mathcal{T}$, characterised by the relation $\ell(\llbracket x, y \rrbracket)=d(x, y)$, for all $x, y \in \mathcal{T}$.

We introduce a Poisson point measure $\mathcal{P}(d t, d x)=\sum_{i>1} \delta_{\left(t_{i}, x_{i}\right)}(d t, d x)$ on $\mathbb{R}_{+} \times \mathcal{T}$ of intensity $d t \ell(d x)$. One can imagine the $\left(t_{i}, x_{i}\right)$ 's as cuts on $\mathcal{T}$ : at time $t_{i}$, the point $x_{i}$ is removed from $\mathcal{T}$, which disconnects the tree. As time moves on, more cuts arrive and $\mathcal{T}$ fragments into finer and finer connected components. The Aldous-Pitman fragmentation consists in describing the time evolution of the collection of $\mu$-masses of these connected components. It is also known that in the case of $k=1$, the size of the subtree of $T_{n}$ containing the root, when suitably rescaled, converges to the $\mu$-mass of the connected component of the CRT containing the root ([4]). Thus, the cutting down of $\mathcal{T}$, which involves removing $x_{i}$ at time $t_{i}$, can be seen as the scaling limit of the $k$-cut model on $T_{n}$ for $k=1$. On the other hand, the key element in our construction is the following time-changed version of $\mathcal{P}$ : for $k \in[1, \infty)$, define

$$
\tilde{\mathcal{P}}=\sum_{i \geq 1} \delta_{\left(s_{i}, x_{i}\right)}, \quad \text { where } \quad s_{i}=\left(\Gamma(k+1) t_{i}\right)^{\frac{1}{k}}, i \geq 1 .
$$

Here, $\Gamma(\cdot)$ is the Gamma function. Let us denote by $\mathcal{T}_{t}=\{x \in \mathcal{T}: \mathcal{P}([0, t] \times \llbracket \rho, x \rrbracket)=0\}$, the subtree connected to the root at time $t$. Similarly, denote $\tilde{\mathcal{T}}_{t}=\{x \in \mathcal{T}: \tilde{\mathcal{P}}([0, t] \times \llbracket \rho, x \rrbracket)=$ 
$0\}$ the remaining subtree in the time-changed cutting process. We define

$$
X_{k}(\mathcal{T})=\int_{0}^{\infty} \mu\left(\tilde{\mathcal{T}}_{t}\right) d t=\int_{0}^{\infty} \mu\left(\mathcal{T}_{t^{k} / \Gamma(k+1)}\right) d t=\frac{(\Gamma(k+1))^{\frac{1}{k}}}{k} \int_{0}^{\infty} \mu\left(\mathcal{T}_{s}\right) s^{\frac{1}{k}-1} d s
$$

For $k=1, X_{1}(\mathcal{T})$ appears in $[5,1,2]$ as the scaling limit of $X_{1}\left(T_{n}\right)$. Let us also recall that Aldous and Pitman [4] have shown that the process $\left(\mu\left(\mathcal{T}_{t}\right)\right)_{t \geq 0}$ has the same distribution as $\left(\left(1+L_{t}\right)^{-1}\right)_{t \geq 0}$ with $\left(L_{t}\right)_{t \geq 0}$ being a $\frac{1}{2}$-stable subordinator. Combined with a Lamperti time-change, this then implies $X_{1}(\mathcal{T})$ has the Rayleigh distribution ([5]). Note that we also have the following bound from (1.3):

$$
k(\Gamma(k+1))^{-\frac{1}{k}} X_{k}(\mathcal{T}) \leq \int_{0}^{1} s^{\frac{1}{k}-1} d s+\int_{1}^{\infty} \mu\left(\mathcal{T}_{s}\right) d s \leq k+X_{1}(\mathcal{T})
$$

So in particular, $X_{k}(\mathcal{T})<\infty$, a.s. Let us also point out that even though the discrete model is only defined for $k \in \mathbb{N}$, the above definition of $X_{k}(\mathcal{T})$ makes sense for all $k \in[1, \infty)$. Here is our main result.

Theorem 1.1. For all $k \in \mathbb{N}$, conditional on $(\mathcal{T}, d), X_{k}(\mathcal{T})$ has the same distribution as $Z_{k}$.

We'll give two proofs of the theorem. In Section 2, we give a first proof by identifying the conditional moments of $X_{k}(\mathcal{T})$ given $\mathcal{T}$ with those of $Z_{k}$, which were computed in [6]. In Section 3, we give a second proof via weak convergence arguments. Even though it takes a bit more space, the second proof is perhaps more helpful in explaining the motivation for the definition (1.3), as well as in providing an alternative proof of the convergence in (1.1).

\section{Conditional expectation of $X_{k}(\mathcal{T})$ given $\mathcal{T}$}

We will need the following notation. For $q \in \mathbb{N}$ and $\mathbf{s}=\left(s_{1}, s_{2}, \ldots, s_{q}\right) \in[0,1]^{q}$, we set $\Delta_{1}^{\mathrm{e}}(\mathbf{s})=e_{s_{1}}$, and more generally for $2 \leq r \leq q$,

$$
\Delta_{r}^{\mathrm{e}}(\mathbf{s})=e_{s_{r}}-\max _{i<r} b\left(s_{i}, s_{r}\right), \quad \text { where } b(s, t)=\min _{s \wedge t \leq u \leq s \vee t} e_{u} .
$$

Note that $\Delta_{1}^{\mathrm{e}}(\mathbf{s})+\cdots+\Delta_{r}^{\mathrm{e}}(\mathbf{s})$ is the total length (i.e. $\ell$-mass) of the reduced subtree of $\mathcal{T}$ spanned by $p\left(s_{1}\right), \ldots, p\left(s_{r}\right)$, for all $r \leq q$. Our goal is to prove the following formulas for the moments of $X_{k}(\mathcal{T})$.

Proposition 2.1. For all $k \geq 1$ and $q \in \mathbb{N}$, we have

$$
\begin{aligned}
\mathbb{E}\left[X_{k}(\mathcal{T})^{q} \mid \mathrm{e}\right]= & q ! \int_{[0,1]^{q}} d s_{1} \cdots d s_{q} \int_{0}^{\infty} \int_{0}^{x_{1}} \cdots \int_{0}^{x_{q-1}} \\
& \exp \left(-\frac{1}{k !}\left(\Delta_{1}^{\mathrm{e}}(\mathbf{s}) x_{1}^{k}+\Delta_{2}^{\mathrm{e}}(\mathbf{s}) x_{2}^{k}+\cdots+\Delta_{q}^{\mathrm{e}}(\mathbf{s}) x_{q}^{k}\right)\right) d x_{q} \cdots d x_{1} .
\end{aligned}
$$

Proof. For $v \in \mathcal{T}$, we define $\mathcal{E}_{v}=\inf \{t>0: \tilde{\mathcal{P}}([0, t] \times \llbracket \rho, v \rrbracket)=1\}$, the moment that $v$ is separated from the root. Then $v \in \tilde{\mathcal{T}}_{t}$ if and only if $\mathcal{E}_{v}>t$. Therefore, we can re-write $X_{k}(\mathcal{T})^{q}$ as follows.

$$
\begin{aligned}
\left(X_{k}(\mathcal{T})\right)^{q} & =\int_{\mathbb{R}_{+}^{q}} \mu\left(\tilde{\mathcal{T}}_{t_{1}}\right) \mu\left(\tilde{\mathcal{T}}_{t_{2}}\right) \cdots \mu\left(\tilde{\mathcal{T}}_{t_{q}}\right) d t_{1} d t_{2} \cdots d t_{q} \\
& =\int_{\mathbb{R}_{+}^{q}} \int_{\mathcal{T}^{q}} \mathbf{1}_{\left\{\mathcal{E}_{v_{1}}>t_{1}, \ldots, \mathcal{E}_{v_{q}}>t_{q}\right\}} \mu\left(d v_{1}\right) \cdots \mu\left(d v_{q}\right) d t_{1} \cdots d t_{q} \\
& =\int_{\mathbb{R}_{+}^{q}} \int_{[0,1]^{q}} \mathbf{1}_{\left\{\mathcal{E}_{p\left(s_{1}\right)}>t_{1}, \ldots, \mathcal{E}_{p\left(s_{q}\right)}>t_{q}\right\}} d s_{1} \cdots d s_{q} d t_{1} \cdots d t_{q}
\end{aligned}
$$




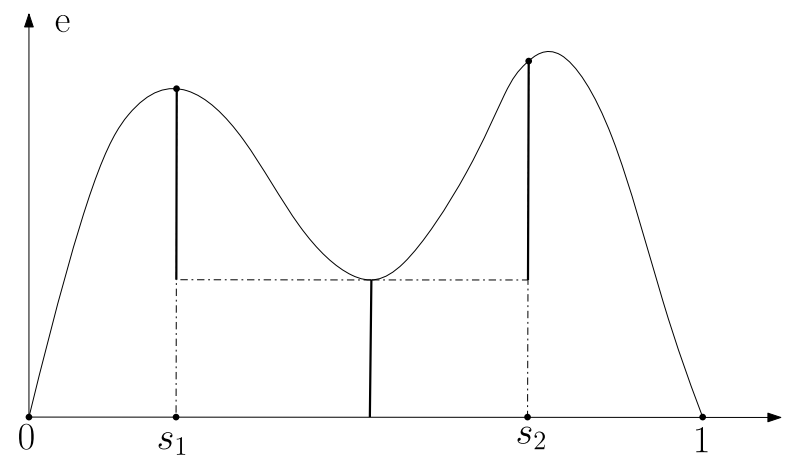

Figure 1: An illustration of $\mathcal{R}_{q}$ with $q=2$. Here, $\mathcal{R}_{q}$ has the shape of a binary tree with 2 leaves, one branch point and three edges (depicted by the line segments in bold). The edge lengths correspond to the lengths of these line segments.

where we have used in the last line the definition that $\mu$ is the push-forward of the Lebesgue measure on $[0,1]$. Write $\mathbb{E}_{\mathrm{e}}$ as a shorthand for $\mathbb{E}[\cdot \mid \mathrm{e}]$. The above yields that

$$
\mathbb{E}_{\mathrm{e}}\left[X_{k}(\mathcal{T})^{q}\right]=\int_{[0,1]^{q}} \int_{\mathbb{R}_{+}^{q}} \mathbb{P}_{\mathrm{e}}\left(\mathcal{E}_{p\left(s_{1}\right)}>t_{1}, \ldots, \mathcal{E}_{p\left(s_{q}\right)}>t_{q}\right) d t_{1} \cdots d t_{q} d s_{1} \cdots d s_{q}
$$

We then split $\mathbb{R}_{+}^{q}$ into $q$ ! subdomains according to the $q$ ! possible rankings of $\left(t_{i}\right)_{1 \leq i \leq q}$. However, $\left(s_{i}\right)_{1 \leq i \leq q}$ is sampled in an i.i.d fashion and is therefore exchangeable, so that integration from each subdomain will contribute equally. Hence,

$$
\begin{aligned}
& \mathbb{E}_{\mathrm{e}}\left[X_{k}(\mathcal{T})^{q}\right] \\
& \quad=q ! \int_{[0,1]^{q}} \int_{0}^{\infty} \int_{0}^{t_{1}} \cdots \int_{0}^{t_{q-1}} \mathbb{P}_{\mathrm{e}}\left(\mathcal{E}_{p\left(s_{1}\right)}>t_{1}, \ldots, \mathcal{E}_{p\left(s_{q}\right)}>t_{q}\right) d t_{q} \cdots d t_{1} d s_{1} \cdots d s_{q} .
\end{aligned}
$$

Let $\mathcal{R}_{q}$ be the reduced subtree of $\mathcal{T}$ spanned by $v_{1}=p\left(s_{1}\right), \ldots, v_{q}=p\left(s_{q}\right)$, i.e. the smallest connected subspace of $\mathcal{T}$ containing these $q$ points and the root $\rho$. Note that $\mathcal{R}_{q}$ is a "finite" tree in the sense that it only has a finite number of branch points and leaves. Here, it will be convenient to think of it as a (graph) tree $\left(\mathrm{V}_{q}, \mathrm{E}_{q}\right)$, where the vertex set $\mathrm{V}_{q}$ consists of the root, the leaves and the branch points of $\mathcal{R}_{q}$ and each edge $e \in \mathrm{E}_{q}$ is equipped with an edge length $l(e) \in(0, \infty)$. These edge lengths are consistent with the distance $d$ in the following way: for each $v \in \mathrm{V}_{q}, d(\rho, v)=\sum_{e \in \mathcal{L}(v)} l(e)$, where $\mathcal{L}(v)$ stands for the set of edges on the path from the root $\rho$ to $v$. See also Fig. 1 for an example of $\mathcal{R}_{q}$. Now to each edge $e$ in this tree, we associate an independent exponential variable $E_{e}$ of mean $1 / l(e)$. It follows from the definition (1.2) of $\tilde{\mathcal{P}}$ that $\left(\mathcal{E}_{v_{r}}\right)^{k} / k$ ! is distributed as an exponential random variable of mean $1 / d\left(\rho, v_{r}\right)=1 / e_{s_{r}}$. It is then straightforward to check that

$$
\left(\mathcal{E}_{v_{r}} ; 1 \leq r \leq q\right) \stackrel{(d)}{=}\left(\min _{e \in \mathcal{L}\left(v_{r}\right)}\left(k ! E_{e}\right)^{1 / k} ; 1 \leq r \leq q\right) .
$$

Bearing in mind that $t_{1}>t_{2}>\cdots>t_{q}$, we then find that

$$
\begin{aligned}
& \mathbb{P}_{\mathrm{e}}\left(\mathcal{E}_{v_{1}}>t_{1}, \ldots, \mathcal{E}_{v_{q}}>t_{q}\right) \\
= & \mathbb{P}_{\mathrm{e}}\left(\left(k ! E_{e}\right)^{\frac{1}{k}}>t_{r}, \forall e \in \mathcal{L}\left(v_{r}\right), 1 \leq r \leq q\right) \\
= & \mathbb{P}_{\mathrm{e}}\left(\left(k ! E_{e}\right)^{\frac{1}{k}}>t_{1}, \forall e \in \mathcal{L}\left(v_{1}\right)\right) \mathbb{P}_{\mathrm{e}}\left(\left(k ! E_{e}\right)^{\frac{1}{k}}>t_{2}, \forall e \in \mathcal{L}\left(v_{2}\right) \backslash \mathcal{L}\left(v_{1}\right)\right) \\
& \cdots \mathbb{P}_{\mathrm{e}}\left(\left(k ! E_{e}\right)^{\frac{1}{k}}>t_{q}, \forall e \in \mathcal{L}\left(v_{q}\right) \backslash \cup_{r<q} \mathcal{L}\left(v_{r}\right)\right) \\
= & \exp \left(-\frac{1}{k !}\left(\Delta_{1}^{\mathrm{e}}(\mathbf{s}) t_{1}^{k}+\Delta_{2}^{\mathrm{e}}(\mathbf{s}) t_{2}^{k}+\cdots \Delta_{q}^{\mathrm{e}}(\mathbf{s}) t_{q}^{k}\right)\right) .
\end{aligned}
$$


By the previous arguments, this completes the proof.

Proof 1 of Theorem 1.1. Comparing (2.1) with equations (8) and (9) in [6], we see that $\mathbb{E}\left[X_{k}(\mathcal{T})^{q} \mid \mathrm{e}\right]=\mathbb{E}\left[Z_{k}^{q} \mid \mathrm{e}\right]$ for all $q \in \mathbb{N}$. Applying Theorem 2 and Lemma 8 there, we conclude that conditional on e, $X_{k}(\mathcal{T})$ has the same distribution as $Z_{k}$.

\section{Scaling limit of $X_{k}\left(T_{n}\right)$}

Here, we give a second proof of the theorem by showing $X_{k}(\mathcal{T})$ is the scaling limit of $X_{k}\left(T_{n}\right)$. Throughout this section, we assume $k \in\{2,3, \ldots\}$.

\subsection{Convergence of random trees}

We briefly recall Aldous' Theorem on the convergence of the conditioned GaltonWatson tree $T_{n}$, as well as provide some necessary background on the Gromov-Hausdorff topology. Further details on these topics can be found in $[13,11,8,15,3]$.

The Gromov-Hausdorff distance between two compact metric spaces $\left(X, d_{X}\right)$ and $\left(Y, d_{Y}\right)$ is the following quantity:

$$
d_{\mathrm{GH}}(X, Y)=\inf _{\phi, \varphi, Z} d_{Z, \text { Haus }}(\phi(X), \varphi(Y))
$$

where the infimum is over all the isometric embeddings $\phi: X \rightarrow Z$ and $\varphi: Y \rightarrow Z$ into a common metric space $\left(Z, d_{Z}\right)$, and $d_{Z \text {, Haus }}$ stands for the usual Hausdorff distance for the compact sets of $Z$. In our application, we often need to keep track of specified points in the initial spaces. To that end, let $\mathbf{x}=\left(x_{1}, \ldots, x_{p}\right)$ and $\mathbf{y}=\left(y_{1}, \ldots, y_{p}\right)$ be $p \in \mathbb{N}$ points of $X$ and $Y$ respectively. Then the marked Gromov-Hausdorff distance between $\left(X, d_{X}, \mathbf{x}\right)$ and $\left(Y, d_{Y}, \mathbf{y}\right)$ is defined as

$$
d_{p, \mathrm{GH}}(X, Y)=\inf _{\phi, \varphi, Z}\left(d_{Z, \mathrm{Haus}}(\phi(X), \varphi(Y)) \vee \max _{1 \leq i \leq p} d_{Z}\left(\phi\left(x_{i}\right), \varphi\left(y_{i}\right)\right)\right),
$$

where the infimum is again over all the isometric embeddings of $X$ and $Y$ into a common metric space. For each $p \geq 1$, it turns out that the space of metric spaces with $p$ marked points is a Polish space with respect to $d_{p, \mathrm{GH}}$ ([15]). Now the convergence of $T_{n}$ mentioned earlier can be given a precise meaning. Let us recall that the Brownian CRT $(\mathcal{T}, d)$ is a metric space by definition. Recall also $\rho \in \mathcal{T}$ stands for its root. Equipping its vertex set with the graph distance, we can also view the tree $T_{n}$ as a metric space. Let us denote by $\frac{\sigma}{\sqrt{n}} T_{n}$ the rescaled metric space where the graph distance is multiplied by a factor $\frac{\sigma}{\sqrt{n}}$. Denote also by $\rho_{n}$ its root. We have

$$
\left(\frac{\sigma}{\sqrt{n}} T_{n}, \rho_{n}\right) \underset{n \rightarrow \infty}{\stackrel{(d)}{\longrightarrow}}(\mathcal{T}, \rho)
$$

in the weak topology of the marked Gromov-Hausdorff distance.

We note that $\mathcal{T}$ is further equipped with a probability measure $\mu$. Let us define its discrete counterpart: for $n \geq 1$, let $\mu_{n}$ be the uniform probability measure on the vertex set of $T_{n}$. In fact, Aldous's Theorem in [3] also implies the following convergence of reduced trees. Given $\mathcal{T}$, let $\left(V_{i}\right)_{i>1}$ be an i.i.d. sequence of points in $\mathcal{T}$ sampled with $\mu$. For $p \in \mathbb{N}$, denote by $\mathcal{R}_{p}$ the reduced tree of $\mathcal{T}$ spanned by $V_{1}, \ldots, V_{p}$. Similarly, we sample an i.i.d. sequence $\left(V_{i}^{n}\right)_{i \geq 1}$ from $T_{n}$ with law $\mu_{n}$. Let $\mathcal{R}_{p}^{n}$ be the reduced subtree of $T_{n}$ spanned by $V_{1}^{n}, \ldots, V_{p}^{n}$, namely, the smallest subgraph of $T_{n}$ (an edge of the subgraph is also an edge of $T_{n}$ ) containing $V_{1}^{n}, \ldots, V_{p}^{n}$ and the root $\rho_{n}$. As above, we denote by $\frac{\sigma}{\sqrt{n}} \mathcal{R}_{p}^{n}$ the metric space obtained from $\mathcal{R}_{p}^{n}$ by equipping its vertex set with $\frac{\sigma}{\sqrt{n}}$ times the graph distance. Then we have

$$
\forall p \in \mathbb{N}, \quad\left(\frac{\sigma}{\sqrt{n}} \mathcal{R}_{p}^{n}, V_{1}^{n}, \ldots, V_{p}^{n}\right) \underset{n \rightarrow \infty}{\stackrel{(d)}{\longrightarrow}}\left(\mathcal{R}_{p}, V_{1}, \ldots, V_{p}\right),
$$


with respect to the marked Gromov-Hausdorff topology. We have seen that $\mathcal{R}_{p}$ can be viewed as a (graph) tree with edge lengths. But so is $\frac{\sigma}{\sqrt{n}} \mathcal{R}_{p}^{n}$, where the edge length is simply $\frac{\sigma}{\sqrt{n}}$. Note that the convergence in (3.2) implies the "shape" of $\mathcal{R}_{p}^{n}$ coincides with that of $\mathcal{R}_{p}$ for large $n$ and

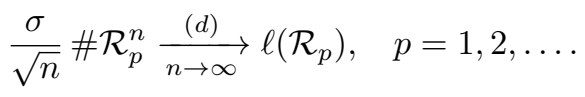

where \# stands for the counting measure on the vertex set of $\mathcal{R}_{p}^{n}$ and $\ell$ is the length measure of $\mathcal{T}$.

Let us recall the Poisson point measure $\mathcal{P}$ has an intensity $d t \ell(d x)$. Since $\ell\left(\mathcal{R}_{p}\right)<\infty$, there is a finite number of cuts $\left(t_{i}, x_{i}\right)$ from $\mathcal{P}$ which fall on $\mathcal{R}_{p}$ before time $t$. So a convenient approach to studying the cutting of $\mathcal{T}$ is first look at those cuts which fall on $\mathcal{R}_{p}, p \geq 1$. We'll also see the convergences in (3.2) and (3.3) will be our starting point for proving the convergence of $X_{k}\left(T_{n}\right)$.

\subsection{Convergence of the cutting process}

For each vertex $v$ of $T_{n}$, let us denote $\eta_{v}=\inf \left\{t: N_{v}(t)=k\right\}$, the time when $v$ is removed from $T_{n}$. We show here that the point measure $\mathcal{P}_{n}:=\sum_{v \in T_{n}} \delta_{\left(\eta_{v}, v\right)}$ converges in an appropriate sense to $\tilde{\mathcal{P}}$. Let us start with the following observation.

Lemma 3.1. For each $m \in \mathbb{N}$, suppose $a_{m} \in(0, \infty)$ and let $\left(G_{m, i}\right)_{1 \leq i \leq m}$ be independent $\operatorname{Gamma}\left(k, \frac{1}{a_{m}}\right)$ random variables whose probability density function is given by $\frac{1}{(k-1) !} a_{m}^{k} x^{k-1} e^{-a_{m} x}, x>0$. Let

$$
\mathcal{N}_{m}(t)=\sum_{1 \leq i \leq m} \mathbf{1}_{\left\{G_{m, i} \leq t\right\}}, \quad t \geq 0
$$

If $m a_{m}^{k} \rightarrow a \in(0, \infty)$ as $m \rightarrow \infty$, then we have

$$
\left(\mathcal{N}_{m}(t)\right)_{t \geq 0} \underset{m \rightarrow \infty}{\stackrel{(d)}{\longrightarrow}}\left(\mathcal{N}\left(t^{k} / k !\right)\right)_{t \geq 0} \quad \text { in } \mathbb{D}\left(\mathbb{R}_{+}, \mathbb{R}\right),
$$

where $(\mathcal{N}(t))_{t \geq 0}$ is a Poisson process on $\mathbb{R}_{+}$of rate $a$ and $\mathbb{D}\left(\mathbb{R}_{+}, \mathbb{R}\right)$ is the space of càdlàg functions endowed with the Skorokhod topology.

Proof. Let $G$ denote a $\operatorname{Gamma}(k, 1)$ random variable and let $X$ be a Poisson random variable of mean $t$. We note that

$$
\mathbb{P}(G \leq t)=\mathbb{P}(X \geq k)=\sum_{j=k}^{\infty} e^{-t} \frac{t^{j}}{j !}=\frac{t^{k}}{k !}+t^{k+1} R(t)
$$

where $R(\cdot)$ is bounded on any finite interval. Let $T>0$. For all $t \leq T$ and $p \geq 0$, noting $\mathbb{P}\left(G_{m, 1} \leq t\right)=\mathbb{P}\left(G \leq a_{m} t\right)$, we deduce that

$$
\begin{aligned}
& \mathbb{P}\left(\mathcal{N}_{m}(t)=p\right) \\
= & \left(\begin{array}{c}
m \\
p
\end{array}\right)\left(\mathbb{P}\left(G_{m, 1} \leq t\right)\right)^{p}\left(\mathbb{P}\left(G_{m, 1}>t\right)\right)^{m-p} \\
= & \frac{\left(\begin{array}{c}
m \\
p
\end{array}\right)}{m^{p}}\left(\frac{m\left(a_{m} t\right)^{k}}{k !}+m\left(a_{m} t\right)^{k+1} R\left(a_{m} t\right)\right)^{p}\left(1-\frac{\left(a_{m} t\right)^{k}}{k !}+\left(a_{m} t\right)^{k+1} R\left(a_{m} t\right)\right)^{m-p} \\
\rightarrow & \frac{1}{p !}\left(\frac{a t^{k}}{k !}\right)^{p} \exp \left(-a t^{k} / k !\right)=\mathbb{P}\left(\mathcal{N}\left(t^{k} / k !\right)=p\right) .
\end{aligned}
$$


We now extend this to multidimensional marginals. Let $l \geq 2,0 \leq t_{1} \leq t_{2} \leq \cdots \leq t_{l}$ and a sequence of non negative integers $p_{1} \leq p_{2} \leq \cdots \leq p_{l}$. Then for $m \geq p_{l}$, we apply (3.4) again to find that

$$
\begin{aligned}
& \mathbb{P}\left(\mathcal{N}_{m}\left(t_{l}\right)=p_{l} \mid \mathcal{N}_{m}\left(t_{i}\right)=p_{i}, 1 \leq i \leq l-1\right) \\
= & \left(\begin{array}{c}
m-p_{l-1} \\
p_{l}-p_{l-1}
\end{array}\right)\left(\mathbb{P}\left(G_{m, 1} \leq t_{l} \mid G_{m, 1}>t_{l-1}\right)\right)^{p_{l}-p_{l-1}}\left(\mathbb{P}\left(G_{m, 1}>t_{l} \mid G_{m, 1}>t_{l-1}\right)\right)^{m-p_{l}} \\
\rightarrow & \frac{1}{\left(p_{l}-p_{l-1}\right) !}\left(\frac{a t_{l}^{k}}{k !}-\frac{a t_{l-1}^{k}}{k !}\right)^{p_{l}-p_{l-1}} \exp \left(-\frac{a\left(t_{l}^{k}-t_{l-1}^{k}\right)}{k !}\right),
\end{aligned}
$$

which is precisely $\mathbb{P}\left(\mathcal{N}\left(t_{l}^{k} / k !\right)=p_{l} \mid \mathcal{N}\left(t_{i}^{k} / k !\right)=p_{i}, 1 \leq i \leq l-1\right)$. Combined with an induction argument, this readily yields the distributional convergence of $\left(\mathcal{N}_{m}\left(t_{i}\right), 1 \leq\right.$ $i \leq l)$ to $\left(\mathcal{N}_{m}\left(t_{i}\right), 1 \leq i \leq l\right)$ for all $\left(t_{i}\right)_{1 \leq i \leq l}, l \geq 1$. Since $t \mapsto \mathcal{N}_{m}(t)$ is non-decreasing, we may conclude that the convergence holds in $\mathbb{D}\left(\mathbb{R}_{+}, \mathbb{R}\right)$.

Recall the reduced trees $\mathcal{R}_{p}^{n}$ and $\mathcal{R}_{p}$. Let us take the vertices $v \in \mathcal{R}_{p}^{n}$ and rank them in the increasing order of the $\eta_{v}$ 's. We write the ranked sequence as $\left(v_{i}^{n, p}\right)_{1 \leq i \leq \# \mathcal{R}_{p}^{n}}$ so that $\eta_{v_{1}^{n, p}}<\eta_{v_{2}^{n, p}}<\cdots<\eta_{v_{\# \mathcal{R}_{p}^{n}}^{n, p}}$. Similarly, since $\tilde{\mathcal{P}}\left([0, t] \times \mathcal{R}_{p}\right)=\#\left\{\left(s_{i}, x_{i}\right): x_{i} \in \mathcal{R}_{p}, s_{i} \leq t\right\}<$ $\infty$ for each $t>0$, we can rank the elements of $\left\{\left(s_{i}, x_{i}\right): x_{i} \in \mathcal{R}_{p}\right\}$ in the increasing order of their first coordinates and write the ranked (infinite) sequence as $\left(\tau_{1}^{p}, \chi_{1}^{p}\right),\left(\tau_{2}^{p}, \chi_{2}^{p}\right), \ldots$ Let us also denote

$$
\delta_{n}=\sigma^{\frac{1}{k}} n^{-\frac{1}{2 k}}, \quad n \geq 1 .
$$

Proposition 3.2. For each $p \geq 1$, as $n \rightarrow \infty$, we have for all $j \geq 1$,

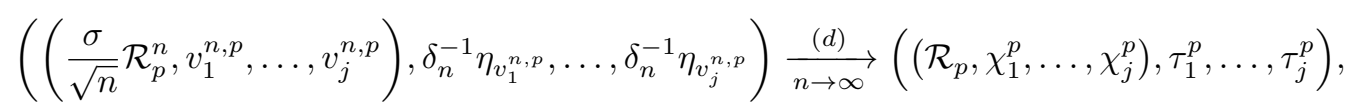

where the convergence of the first coordinates is with respect to the marked GromovHausdorff topology.

Proof. Since the $\eta_{v}$ 's are i.i.d, the law of $\left(v_{1}^{n, p}, \ldots, v_{j}^{n, p}\right)$ is that of a uniform sample without replacement of size $j$ from $\mathcal{R}_{p}$, and is further independent of $\left(\eta_{v_{i}^{n, p}}\right)_{1 \leq i \leq j}$. Combined with the convergence in (3.2), this implies that $\left(v_{1}^{n, p}, \ldots, v_{j}^{n, p}\right)$ converges in distribution to $j$ independent uniform points in $\mathcal{R}_{p}$, which is precisely the distribution of $\chi_{1}^{p}, \ldots, \chi_{j}^{p}$. So it remains to check the convergence of $\eta_{v_{i}^{n, p}}$. Let us define

$$
\mathcal{N}_{n, p}(t)=\sum_{v \in \mathcal{R}_{p}^{n}} \mathbf{1}_{\left\{\eta_{v} \leq \delta_{n} t\right\}}=\max \left\{i: \eta_{v_{i}^{n, p}} \leq \delta_{n} t\right\}, \quad t \geq 0 .
$$

Since each $\delta_{n}^{-1} \eta_{v}$ is distributed as an independent $\operatorname{Gamma}\left(k, \frac{1}{\delta_{n}}\right)$, applying Lemma 3.1 with $m=\# \mathcal{R}_{p}^{n}$ and $a_{m}=\delta_{n}$, we obtain from (3.3) that $\left(\mathcal{N}_{n, p}^{\delta_{n}}(t)\right)_{t \geq 0}$ converges in distribution to $\mathcal{N}\left(t^{k} / k !\right)_{t \geq 0}$, a Poisson process of rate $\ell\left(\mathcal{R}_{p}\right)$. By (1.2), the latter has the same law as $\left(\tilde{\mathcal{P}}\left([0, t] \times \mathcal{R}_{p}\right)\right)_{t \geq 0}$. Since the law of $\left(\eta_{v_{i}^{n, p}}^{n}\right)_{1 \leq i \leq j}$ is determined by $\left(\mathcal{N}_{n, p}(t)\right)_{t \geq 0}$ in the same way that of $\left(\tau_{i}^{p}\right)_{1 \leq i \leq j}$ is by $\left(\mathcal{N}\left(t^{k} / k !\right)\right)_{t \geq 0}$, this completes the proof.

Let $T_{n}(t)$ be the subtree of $T_{n}$ formed by the vertices connected to the root at time $t$. Note that a vertex $v \in T_{n}(t)$ if and only if none of its ancestors nor $v$ itself has been removed by time $t$. Let us denote $\mu_{n}(t)=\mu_{n}\left(T_{n}(t)\right)$. Recall that $\tilde{\mathcal{T}}_{t}$ is the subtree of $\mathcal{T}$ connected to the root at time $t$ from the cutting process $\tilde{\mathcal{P}}$. Proposition 3.2 implies the following

Lemma 3.3. As $n \rightarrow \infty$, jointly with the convergence in (3.2), we have that $\left(\mu_{n}\left(\delta_{n} t\right)\right)_{t \geq 0}$ converges to $\left(\mu\left(\tilde{\mathcal{T}}_{t}\right)\right)_{t \geq 0}$ in distribution with respect to the Skorokhod topology on $\mathrm{D}\left(\mathbb{R}_{+}, \mathbb{R}\right)$. 
Proof. The arguments are similar to the ones in Section 2.3, [4], so we'll only sketch the proof. Recall that $\left(V_{i}^{n}\right)_{i \geq 1}$ (resp. $\left(V_{i}\right)_{i \geq 1}$ ) is a sequence of i.i.d. uniform vertices of $T_{n}$ (resp. i.i.d. points of $\mathcal{T}$ with law $\mu$ ). By the Strong Law of Large Numbers, we have for each $t>0$,

$$
\frac{1}{j} \sum_{i=1}^{j} \mathbf{1}_{\left\{V_{i}^{n} \in T_{n}(t)\right\}} \underset{j \rightarrow \infty}{\stackrel{\text { a.s. }}{\longrightarrow}} \mu_{n}(t) \quad \text { and } \quad \frac{1}{j} \sum_{i=1}^{j} \mathbf{1}_{\left\{V_{i} \in \tilde{\mathcal{T}}_{t}\right\}} \underset{j \rightarrow \infty}{\stackrel{\text { a.s. }}{\longrightarrow}} \mu\left(\tilde{\mathcal{T}}_{t}\right) .
$$

On the other hand, $V_{i}^{n} \in T_{n}(t)$ if and only if the first $\eta_{v}$ for those $v$ in the path from the root to $V_{j}^{n}$ arrives after $t$. Therefore, according to Proposition 3.2 , for each $j \geq 1$,

$$
\left(\mathbf{1}_{\left\{V_{i}^{n} \in T_{n}\left(\delta_{n} t\right)\right\}}, 1 \leq i \leq j\right) \underset{n \rightarrow \infty}{\stackrel{(d)}{\longrightarrow}}\left(\mathbf{1}_{\left\{V_{i} \in \mathcal{T}(t)\right\}}, 1 \leq i \leq j\right)
$$

It follows that we can find a sequence $k_{n} \rightarrow \infty$ slowly enough such that

$$
\frac{1}{k_{n}} \sum_{i=1}^{k_{n}} \mathbf{1}_{\left\{V_{i}^{n} \in T_{n}\left(\delta_{n} t\right)\right\}}^{\stackrel{(d)}{\longrightarrow \rightarrow \infty}} \mu\left(\tilde{\mathcal{T}}_{t}\right)
$$

jointly with (3.2). Invoking the Strong Law of Large Numbers again, we deduce that $\mu_{n}\left(\delta_{n} t\right) \rightarrow \mu\left(\tilde{\mathcal{T}}_{t}\right)$ in distribution, jointly with (3.2). These arguments can also be adapted to prove the convergence of the multidimentional marginals. The functional convergence then follows thanks to monotonicity.

By Skorokhod's representation theorem, we can assume from now on that jointly with (3.2), we have

$$
\left(\mu_{n}\left(\delta_{n} t\right)\right)_{t \geq 0} \stackrel{n \rightarrow \infty}{\longrightarrow}\left(\mu\left(\tilde{\mathcal{T}}_{t}\right)\right)_{t \geq 0} \text { a.s. in } \mathbb{D}\left(\mathbb{R}_{+}, \mathbb{R}\right) .
$$

\subsection{Records and numbers of "cuts"}

Recall the Poisson process $N_{v}$ associated to each vertex $v \in T_{n}$. Let us write $\eta_{v, r}=\inf \left\{t: N_{v}(t)=r\right\}$ for the $r$-th jump of $N_{v}$; in particular, $\eta_{v, k}=\eta_{v}$. For $r=1, \cdots, k$, we say $v$ is a $r$-record if $v$ is still connected to the root at time $\eta_{v, r}$. Denote by $X_{k, r}\left(T_{n}\right)$ the total number of $r$-records in $T_{n}$. Clearly, $X_{k}\left(T_{n}\right)=\sum_{1 \leq r \leq k} X_{k, r}\left(T_{n}\right)$.

To find the scaling limiting of $X_{k, r}\left(T_{n}\right)$, let us introduce $a_{n, r}(t)=\#\left\{v \in T_{n}(t)\right.$ : $\left.N_{v}(t)=r-1\right\}, r=1,2, \ldots, k$. Standard tools from stochastic analysis yield the following

Lemma 3.4. For all $n \geq 1$ and $r \in\{1,2, \ldots, k\}$, we have

$$
\mathbb{E}\left[X_{k, r}\left(T_{n}\right)\right]=\mathbb{E}\left[\int_{0}^{\infty} a_{n, r}(t) d t\right]
$$

and

$$
\mathbb{E}\left[\left(X_{k, r}\left(T_{n}\right)-\int_{0}^{\infty} a_{n, r}(t) d t\right)^{2}\right]=\mathbb{E}\left[\int_{0}^{\infty} a_{n, r}(t) d t\right] .
$$

Proof. For $t>0$, let us denote

$$
\mathcal{X}_{n, r}(t)=\sum_{v \in T_{n}} \mathbf{1}_{\left\{\eta_{v, r} \leq t\right\}} \mathbf{1}_{\{v \text { is an } r \text {-record }\}},
$$

the number of $r$-records which have occurred by time $t$. Clearly, $\mathcal{X}_{n, r}(\infty)=X_{k, r}\left(T_{n}\right)$. Note that given $\eta_{v, r-1}<t<\eta_{v, r}, \eta_{v, r}-t$ is distributed as an exponential variable with mean 1 , with the convention that $\eta_{v, 0}=0$. It is then classical that

$$
M_{r}(t)=\mathcal{X}_{n, r}(t)-\int_{0}^{t} a_{n, r}(s) d s, \quad t \geq 0
$$


is a martingale which further satisfies that $\mathbb{E}\left[M_{r}(t)^{2}\right]=\mathbb{E}\left[\int_{0}^{t} a_{n, r}(s) d s\right]$. In the terminology of point processes, this is saying that $\left(\int_{0}^{t} a_{n, r}(s) d s\right)_{t \geq 0}$ is the compensator of $\left(\mathcal{X}_{n, r}(t)\right)_{t \geq 0}$. On the other hand, for each fixed $n$, one can easily convince oneself that $\mathbb{E}\left[\int_{0}^{\infty} a_{n, r}(s) d s\right]<\infty$. Therefore, $\left(M_{r}(t)\right)_{t \geq 0}$ is also bounded in $L^{2}$. Taking $t \rightarrow \infty$ yields the desired result.

Lemma 3.5. For each $t>0$, we have $\frac{1}{n} a_{n, 1}\left(\delta_{n} t\right)-\mu_{n}\left(\delta_{n} t\right) \rightarrow 0$ in $L^{1}$ and $\mathbb{E}\left[a_{n, r}\left(\delta_{n} t\right)\right] \leq$ $n\left(\delta_{n} t\right)^{r-1}$, for $r \in\{2,3, \ldots, k\}$.

Proof. Conditional on $\mu_{n}(t), a_{n, r}(t)$ is distributed as $\operatorname{Binomial}\left(n \mu_{n}(t), e^{-t} t^{r-1} /(r-1)\right.$ !). Hence,

$$
\mathbb{E}\left[\left|\frac{1}{n} a_{n, 1}\left(\delta_{n} t\right)-\mu_{n}\left(\delta_{n} t\right)\right|\right]=\mathbb{E}\left[\mu_{n}\left(\delta_{n} t\right)\right]\left(1-e^{-\delta_{n} t}\right) \leq \delta_{n} t \rightarrow 0, \quad \text { as } n \rightarrow \infty .
$$

Similarly, we have $\mathbb{E}\left[a_{n, r}\left(\delta_{n} t\right)\right] \leq \mathbb{E}\left[n \mu_{n}\left(\delta_{n} t\right)\right]\left(\delta_{n} t\right)^{r-1} \leq n\left(\delta_{n} t\right)^{r-1}$.

Lemma 3.6. We have

$$
\lim _{t \rightarrow \infty} \limsup _{n \rightarrow \infty} \mathbb{E}\left[\int_{t}^{\infty} \mu_{n}\left(\delta_{n} s\right) d s\right]=0 .
$$

Proof. The first part of the proof is identical to that of Lemma 3 in [5]. We include it here for the sake of completeness. Let $p(t)=\mathbb{P}\left(\eta_{v}>t\right)$ be the probability that $v$ is not removed at time $t$. We note that $v \in T_{n}(t)$ if and only if $\eta_{w}>t$, for every vertex $w$ in the path from the root to $v$. Letting $h t(v)$ be the number of vertices in that path, we can write

$$
\mathbb{E}\left[n \mu_{n}(t)\right]=\mathbb{E}\left[\sum_{v \in T_{n}} \mathbb{P}\left(v \in T_{n}(t) \mid T_{n}\right)\right]=\mathbb{E}\left[\sum_{v \in T_{n}} p(t)^{\mathrm{ht}(v)}\right]=\sum_{m \geq 1} p(t)^{m} \mathbb{E}\left[Q_{m}\left(T_{n}\right)\right],
$$

where $Q_{m}\left(T_{n}\right)=\#\left\{v \in T_{n}: \operatorname{ht}(v)=m\right\}$. Now according to Theorem 1.13 in [12], there exists some constant $C \in(0, \infty)$ which only depends on the offspring distribution $\xi$ such that $\mathbb{E}\left[Q_{m}\left(T_{n}\right)\right] \leq C m$ for all $n$ and $m$. It follows that

$$
n \mathbb{E}\left[\mu_{n}(t)\right] \leq C \sum_{m \geq 1} m p(t)^{m}=\frac{C p(t)}{(1-p(t))^{2}} .
$$

On the other hand, since $\eta_{v}$ has the same distribution as the sum of $k$ independent exponential variables of mean 1 , we deduce the bound $p(t) \leq k \exp (-t / k)$. For small values of $t$, we will use instead:

$$
1-p(t)=\mathbb{P}\left(\eta_{v} \leq t\right)=\int_{0}^{t} \frac{s^{k-1}}{(k-1) !} e^{-s} d s \geq e^{-t} \int_{0}^{t} \frac{s^{k-1}}{(k-1) !} d s=\frac{t^{k}}{k !} e^{-t}, \quad t \geq 0 .
$$

Let $t_{0}$ be such that $k \exp \left(-t_{0} / k\right)<1$. Applying the previous bounds, we find that for $n$ large enough,

$$
\begin{aligned}
\mathbb{E}\left[\int_{t}^{\infty} \mu_{n}\left(\delta_{n} s\right) d s\right] & \leq \frac{C}{n} \int_{t}^{\infty} \frac{p\left(\delta_{n} s\right)}{\left(1-p\left(\delta_{n} s\right)\right)^{2}} \\
& \leq \frac{C}{n} \int_{t}^{t_{0} / \delta_{n}} \frac{d s}{e^{-2 \delta_{n} s}\left(\delta_{n} s\right)^{2 k} /(k !)^{2}}+\frac{C}{n} \int_{t_{0} / \delta_{n}}^{\infty} \frac{k e^{-\delta_{n} s / k}}{\left(1-k e^{-\delta_{n} s / k}\right)^{2}} d s \\
& \leq \frac{C(k !)^{2} e^{2 t_{0}}}{n \delta_{n}^{2 k}} t^{-2 k+1}+\frac{C k^{2}}{n \delta_{n}} \frac{e^{-t_{0} / k}}{1-k e^{-t_{0} / k}}
\end{aligned}
$$

where we have used a change of variable $u=k e^{-\delta_{n} t / k}$ to compute the integral over $\left[t_{0} / \delta_{n}, \infty\right)$. Since $n \delta_{n}^{2 k}=\sigma^{2}$ and $n \delta_{n} \rightarrow \infty$, the conclusion follows. 
Proposition 3.7. As $n \rightarrow \infty$, we have the joint convergence

$$
\left(\frac{\sigma}{\sqrt{n}} T_{n}, \frac{1}{n \delta_{n}} X_{k}\left(T_{n}\right)\right) \stackrel{(d)}{\longrightarrow}\left(\mathcal{T}, X_{k}(\mathcal{T})\right),
$$

where the convergence of the first coordinate is in the Gromov-Hausdorff sense.

Proof. We first note that $\mathbb{E}\left[X_{k}(\mathcal{T})\right]<\infty$ as a consequence of (1.4) and the fact that a Rayleigh distribution has finite mean. Together with Lemma 3.6, this implies that for $\epsilon>0$, we can find $t_{0}=t_{0}(\epsilon) \in(0, \infty)$ such that

$$
\mathbb{E}\left[\int_{t_{0}}^{\infty} \mu\left(\tilde{\mathcal{T}}_{t}\right) d t\right]<\epsilon \text { and } \mathbb{E}\left[\int_{t_{0}}^{\infty} \frac{1}{n} a_{n}\left(\delta_{n} t\right) d t\right] \leq \mathbb{E}\left[\int_{t_{0}}^{\infty} \mu_{n}\left(\delta_{n} t\right) d t\right]<\epsilon, \text { for all } n \geq 1 .
$$

Let $m \in \mathbb{N}$ and take $M \in \mathbb{N}$ large enough such that $M 2^{-m} \geq t_{0}$. Since $t \mapsto a_{n}(t)$ is non increasing, we have

$$
2^{-m} \sum_{j=1}^{M} a_{n}\left(\frac{j \delta_{n}}{2^{m}}\right) \leq \int_{0}^{M / 2^{m}} a_{n}\left(\delta_{n} t\right) d t=\sum_{j=1}^{M} \int_{(j-1) / 2^{m}}^{j / 2^{m}} a_{n}\left(\delta_{n} t\right) d t \leq 2^{-m} \sum_{j=1}^{M} a_{n}\left(\frac{(j-1) \delta_{n}}{2^{m}}\right) .
$$

Replacing $a_{n}\left(\delta_{n} t\right)$ with $\mu\left(\tilde{\mathcal{T}}_{t}\right)$ yields a similar bound for $\int_{0}^{M / 2^{m}} \mu\left(\tilde{\mathcal{T}}_{t}\right) d t$. Then,

$$
\begin{aligned}
\mid \int_{0}^{M / 2^{m}} & \frac{1}{n} a_{n}\left(\delta_{n} t\right) d t-\int_{0}^{M / 2^{m}} \mu\left(\tilde{\mathcal{T}}_{t}\right) d t\left|\leq 2^{-m+1}+2^{-m+1} \sum_{j=1}^{M}\right| \frac{1}{n} a_{n}\left(\frac{j \delta_{n}}{2^{m}}\right)-\mu\left(\tilde{\mathcal{T}}_{\frac{j}{2^{m}}}\right) \mid \\
& \leq 2^{-m+1}+2^{-m+1} \sum_{j=1}^{M}\left\{\left|\frac{1}{n} a_{n}\left(\frac{j \delta_{n}}{2^{m}}\right)-\mu_{n}\left(\frac{j \delta_{n}}{2^{m}}\right)\right|+\left|\mu_{n}\left(\frac{j \delta_{n}}{2^{m}}\right)-\mu\left(\tilde{\mathcal{T}}_{\frac{j}{2^{m}}}\right)\right|\right\} .
\end{aligned}
$$

As a consequence of Lemma 3.5 and (3.5), we obtain

$$
\mathbb{P}\left(\limsup _{n \rightarrow \infty}\left|\int_{0}^{M / 2^{m}} \frac{1}{n} a_{n}\left(\delta_{n} t\right) d t-\int_{0}^{M / 2^{m}} \mu\left(\tilde{\mathcal{T}}_{t}\right) d t\right|>2^{-m+1}\right) \rightarrow 0, \quad \text { as } n \rightarrow \infty,
$$

jointly with the convergences in (3.2). On the other hand, Lemma 3.4 and a change of variable yield

$$
\mathbb{E}\left[\left(\frac{X_{k, 1}\left(T_{n}\right)}{n \delta_{n}}-\int_{0}^{\infty} \frac{1}{n} a_{n}\left(\delta_{n} t\right) d t\right)^{2}\right]=\frac{1}{n \delta_{n}} \mathbb{E}\left[\int_{0}^{\infty} a_{n}(t) d t\right] \rightarrow 0
$$

as $n \rightarrow \infty$. Combining this with (3.9) and (3.8), we obtain

$$
\frac{1}{n \delta_{n}} X_{k, 1}\left(T_{n}\right) \stackrel{n \rightarrow \infty}{\longrightarrow} X_{k}(\mathcal{T}) \quad \text { in probability, }
$$

jointly with the convergences in (3.2). On the other hand, for $r=2,3, \ldots, k$, appealing to Lemma 3.4 and Lemma 3.5, we find that

$$
\frac{\mathbb{E}\left[X_{k, r}\left(T_{n}\right)\right]}{n \delta_{n}}=\frac{1}{n} \mathbb{E}\left[\int_{0}^{\infty} a_{n, r}\left(\delta_{n} t\right) d t\right] \leq t_{0}\left(\delta_{n} t_{0}\right)^{r-1}+\mathbb{E}\left[\int_{t_{0}}^{\infty} \frac{1}{n} a_{n}\left(\delta_{n} t\right) d t\right] .
$$

It follows that $\frac{1}{n \delta_{n}} X_{k, r}\left(T_{n}\right) \rightarrow 0$ in probability by (3.8) and Markov's inequality. Since $X_{k}\left(T_{n}\right)=\sum_{1 \leq r \leq k} X_{k, r}\left(T_{n}\right)$, we deduce from (3.10) that

$$
\frac{1}{n \delta_{n}} X_{k}\left(T_{n}\right) \stackrel{n \rightarrow \infty}{\longrightarrow} X_{k}(\mathcal{T}) \quad \text { in probability }
$$

jointly with the convergences in (3.2). Combined with (3.1), this shows the convergence of both marginals in (3.7). To get to the joint convergence, it suffices to note that the law of $\left(\mathcal{T}, X_{k}(\mathcal{T})\right)$ is the unique limit point of those on the left-hand side, which follows from the joint convergence in (3.11) and the fact that the family $\left(\mathcal{R}_{p}\right)_{p \geq 1}$ uniquely determines the law of $(\mathcal{T}, d, \mu)$. 
$k$-cut model

Proof 2 of Theorem 1.1. This follows by comparing the convergence in Proposition 3.7 with (1.1).

\section{References}

[1] Romain Abraham and Jean-François Delmas, Record process on the continuum random tree, ALEA Lat. Am. J. Probab. Math. Stat. 10 (2013), no. 1, 225-251. MR-3083925

[2] Louigi Addario-Berry, Nicolas Broutin, and Cecilia Holmgren, Cutting down trees with a Markov chainsaw, Ann. Appl. Probab. 24 (2014), no. 6, 2297-2339. MR-3262504

[3] David Aldous, The continuum random tree. III, Ann. Probab. 21 (1993), no. 1, 248-289. MR-1207226

[4] David Aldous and Jim Pitman, The standard additive coalescent, Ann. Probab. 26 (1998), no. 4, 1703-1726. MR-1675063

[5] Jean Bertoin and Grégory Miermont, The cut-tree of large Galton-Watson trees and the Brownian CRT, Ann. Appl. Probab. 23 (2013), no. 4, 1469-1493. MR-3098439

[6] Gabriel Berzunza, Xing Shi Cai, and Cecilia Holmgren, The $k$-cut model in deterministic and random trees, Electron. J. Combin. 28 (2021), no. 1, Paper No. 1.25, 30 pp. MR-4245258

[7] Nicolas Broutin and Minmin Wang, Cutting down p-trees and inhomogeneous continuum random trees, Bernoulli 23 (2017), no. 4A, 2380-2433. MR-3648034

[8] Dmitri Burago, Yuri Burago, and Sergei Ivanov, A course in metric geometry, Graduate Studies in Mathematics, vol. 33, American Mathematical Society, Providence, RI, 2001. MR-1835418

[9] Xing Shi Cai, Cecilia Holmgren, Luc Devroye, and Fiona Skerman, $k$-cut on paths and some trees, Electron. J. Probab. (2019), no. 53, 1-22. MR-3968715

[10] Daphné Dieuleveut, The vertex-cut-tree of Galton-Watson trees converging to a stable tree, Ann. Appl. Probab. 25 (2015), no. 4, 2215-2262. MR-3349006

[11] Steven N. Evans, Probability and real trees, Lecture Notes in Mathematics, vol. 1920, Springer, Berlin, 2008. MR-2351587

[12] Svante Janson, Random cutting and records in deterministic and random trees, Random Structures Algorithms 29 (2006), no. 2, 139-179. MR-2245498

[13] Jean-François Le Gall, Random trees and applications, Probab. Surv. 2 (2005), 245-311. MR-2203728

[14] A. Meir and J. W. Moon, Cutting down random trees, J. Austral. Math. Soc. 11 (1970), 313-324. MR-0284370

[15] Grégory Miermont, Tessellations of random maps of arbitrary genus, Ann. Sci. Éc. Norm. Supér. (4) 42 (2009), no. 5, 725-781. MR-2571957

Acknowledgments. I am thankful to the anonymous referee and the editor for helpful suggestions. 


\section{Electronic Journal of Probability Electronic Communications in Probability}

\section{Advantages of publishing in EJP-ECP}

- Very high standards

- Free for authors, free for readers

- Quick publication (no backlog)

- Secure publication $\left(\mathrm{LOCKSS}^{1}\right)$

- Easy interface (EJMS²)

\section{Economical model of EJP-ECP}

- Non profit, sponsored by $\mathrm{IMS}^{3}, \mathrm{BS}^{4}$, ProjectEuclid ${ }^{5}$

- Purely electronic

\section{Help keep the journal free and vigorous}

- Donate to the IMS open access fund ${ }^{6}$ (click here to donate!)

- Submit your best articles to EJP-ECP

- Choose EJP-ECP over for-profit journals

\footnotetext{
${ }^{1}$ LOCKSS: Lots of Copies Keep Stuff Safe http://www. lockss.org/

${ }^{2}$ EJMS: Electronic Journal Management System http://www.vtex.lt/en/ejms.html

${ }^{3}$ IMS: Institute of Mathematical Statistics http://www.imstat.org/

${ }^{4}$ BS: Bernoulli Society http://www. bernoulli-society.org/

${ }^{5}$ Project Euclid: https://projecteuclid.org/

${ }^{6}$ IMS Open Access Fund: http://www.imstat.org/publications/open.htm
} 